\title{
A developmental language disorder might increase the risk of reoffending
}

By Dr. Jessica Edwards

Researchers in the UK are the first to identify the potential impact of a developmental language disorder (DLD) on reoffending risk in young people. Maxine Winstanley and colleagues recruited 145 young offenders to their study. They collected data on expressive and receptive language, nonverbal IQ and callous-unemotional traits. They then examined differences in the risk of reoffending between young offenders with and without a DLD.

The researchers found that young offenders with a DLD were more than 2.5 times as likely to reoffend within a year of a receiving a court order compared to those without a DLD. This increased risk was not explained by differences in nonverbal IQ, age at first offence, the number of previous offences, a composite adversity score, deprivation score, the presence of a neurodevelopmental disorder or callous-unemotional traits.

Winstanley et al. consider that language could be a "key factor in the continuation of reoffending behaviour". As such, they propose that young people with an unidentified DLD represent a group who are "challenged in their ability to access verbally mediated strategies in the youth justice service". Going forward, it seems important to identify youths with a DLD upon their entry into the youth justice system.

\section{Referring to:}

Winstanley, M., Webb, R.T. \& ContiRamsden, G. (2020), Developmental language disorders and risk of recidivism among young offenders. J. Child Psychol. Psychiatr. doi: 10.1111/ jcpp.13299.

\section{See also:}

Other research digests on

Developmental Language Disorders can be found via the following link:

https://www.acamh.org/researchcollection/developmentallanguage-disorder/

\section{Glossary:}

\section{Developmental language disorder} (DLD): a DLD is diagnosed when a child's language skills are persistently below the level expected for the child's age and this impacts on their everyday life. 